\title{
Allogeneic Most Closely HLA-matched Adenovirus-specific Cytotoxic T Lymphocytes
}

National Cancer Institute

\section{Source}

National Cancer Institute. Allogeneic Most Closely HLA-matched Adenovirus-specific

Cytotoxic T Lymphocytes. NCI Thesaurus. Code C150553.

A population of off the shelf, closely human leukocyte antigen (HLA)-matched allogeneic, ex vivo-expanded cytotoxic T-lymphocytes (CT Ls) specifically reactive to human adenovirus (Ad), with potential immunomodulating and anti-adenoviral activities. Upon administration, the allogeneic most closely HLA-matched Ad-specific CT Ls may reconstitute Ad-specific CTL responses in patients at risk of developing Ad infections either following allogeneic stem cell transplantation or in Ad-infected immunocompromised hosts. The anti-adenoviral CT Ls are provided by a third party donor and not by the allogeneic stem cell transplant donor. 